\title{
Student difficulties regarding symbolic and graphical representations of vector fields
}

\author{
Laurens Bollen, ${ }^{1, *}$ Paul van Kampen, ${ }^{2, \dagger}$ Charles Baily, ${ }^{3, \ddagger}$ Mossy Kelly, ${ }^{4, \S}$ and Mieke De Cock ${ }^{1, \|}$ \\ ${ }^{1}$ KU Leuven, Department of Physics and Astronomy \& LESEC, \\ Celestijnenlaan 200c, 3001 Leuven, Belgium \\ ${ }^{2}$ Centre for the Advancement of STEM Teaching and Learning \& School of Physical Sciences, \\ Dublin City University, Glasnevin, Dublin 9, Ireland \\ ${ }^{3}$ School of Physics \& Astronomy, University of St Andrews, St Andrews, KY16 9SS, United Kingdom \\ ${ }^{4}$ Department of Physics and Mathematics, University of Hull, \\ Kingston-upon-Hull, HU6 7RX, United Kingdom \\ (Received 5 April 2017; published 16 August 2017)
}

\begin{abstract}
The ability to switch between various representations is an invaluable problem-solving skill in physics. In addition, research has shown that using multiple representations can greatly enhance a person's understanding of mathematical and physical concepts. This paper describes a study of student difficulties regarding interpreting, constructing, and switching between representations of vector fields, using both qualitative and quantitative methods. We first identified to what extent students are fluent with the use of field vector plots, field line diagrams, and symbolic expressions of vector fields by conducting individual student interviews and analyzing in-class student activities. Based on those findings, we designed the Vector Field Representations test, a free response assessment tool that has been given to 196 second- and third-year physics, mathematics, and engineering students from four different universities. From the obtained results we gained a comprehensive overview of typical errors that students make when switching between vector field representations. In addition, the study allowed us to determine the relative prevalence of the observed difficulties. Although the results varied greatly between institutions, a general trend revealed that many students struggle with vector addition, fail to recognize the field line density as an indication of the magnitude of the field, confuse characteristics of field lines and equipotential lines, and do not choose the appropriate coordinate system when writing out mathematical expressions of vector fields.
\end{abstract}

DOI: 10.1103/PhysRevPhysEducRes.13.020109

\section{INTRODUCTION}

Vectors play a prominent role in many branches of physics. A vector is a geometric object that has a magnitude (sometimes called length) and direction, and is often visualized as an arrow. Force, momentum, displacement, velocity, acceleration, or torque are just a few examples of physical quantities that are represented by a vector. When a vector is assigned to every point in a subset of space, the resulting structure is called a vector field. A threedimensional vector field has three components, which may depend on three spatial coordinates each (e.g., $\mathbf{v}=y \hat{\mathbf{x}}$ has an $x$ component that depends on the $y$ coordinate). Numerous examples of vector fields can be found in physics, for example, the velocity field of a moving fluid,

\footnotetext{
*Laurens.Bollen@kuleuven.be

†aul.van.Kampen@dcu.ie

crb6@st-andrews.ac.uk

\$Mossy.Kelly@hull.ac.uk

"Mieke.DeCock@kuleuven.be
}

Published by the American Physical Society under the terms of the Creative Commons Attribution 4.0 International license. Further distribution of this work must maintain attribution to the author(s) and the published article's title, journal citation, and DOI.
Newton's gravitational field, or the electromagnetic fields that were described by Maxwell.

A mathematics or physics concept or problem can be expressed in many different ways. It can be a completely textual description of a situation, a symbolic expression, a graph, a picture, a diagram, and so on. These different formats are called representations. Vector fields can be represented by a symbolic (algebraic) expression, using unit vectors of a chosen coordinate system. Furthermore, vector fields are often expressed in a graphical representation, using either a field vector plot or a field line diagram. In a field vector plot, a vector field is visualized as a collection of arrows placed at representative points, each of them with its own direction and length (magnitude). The location of the vector corresponds to the tail of the arrow [1]. A field line is a continuous line drawn so that the tangent to each point on the line is along the direction of the field vector at that point. The lines themselves give information about the direction of the field, and their density indicates the magnitude of the field over a given region. The field line representation therefore inherently interprets a vector as a flux density; this dual meaning is exemplified by the $\mathbf{B}$-field being called both "magnetic field" and "magnetic flux density." Field lines must start at a point with positive divergence and must end at a point with negative divergence. 
In this work, we discuss students' understanding of the aforementioned representations of vector fields in a mathematical context, and their ability to switch between symbolic expressions, field vector plots, and field line diagrams. Abilities related to interpreting various representations and switching between them are considered to be very important in problem solving [2,3]. In addition, using multiple representations is generally accepted to be an effective instructional approach when aiming to improve students'understanding of a certain concept or operation [4-12]. However, learners often fail to exploit the advantages of multiple representations [13-15], or may struggle to switch between them $[3,16]$.

Adopting the terminology used by De Cock [17] and Nistal et al. $[18,19]$, we distinguish between two skills that are needed to benefit from using multiple representations in physics: representational fluency and representational flexibility. Fluency refers to the ability to construct or interpret certain representations like equations, diagrams, or graphs, but also to what extent someone can switch between different representations on demand $[19,20]$. Representational flexibility involves making appropriate representational choices when solving problems [18,19,21]. The work described in this paper focuses solely on students'representational fluency considering vector fields.

The importance of using visualizations in physics has been researched extensively. Studies by Nguyen et al. [22-25] pointed out that many traditional mathematics and physics courses focus insufficiently on graphical representations. In addition, they argued that representational difficulties often arise because students do not activate the appropriate mathematical knowledge. In the context of vector fields, Gire and Price [26] discussed how various representations can be used when teaching vector calculus. Based on their experience with different types of in-class activities, they argued that algebraic representations are useful since they can easily be manipulated, but students gain more insight into the differences between components and coordinates when using a graphical approach.

Cao and Brizuela [27] investigated how high school students aged 15 to 16 years represent electric fields before instruction, and found out that some of them sketched arrows that are very similar to field lines. Nevertheless, some of the features of field lines were misunderstood. Sağlam and Millar [28] reported that high school students misinterpreted magnetic field lines as representing a "flow" from the magnetic north pole to the magnetic south pole, similar to how electric currents flow. In addition, Albe et al. [29] observed that field line diagrams that are sketched by students are often meaningless because they do not reflect important features like the magnitude or direction of the field. Belcher et al. $[30,31]$ developed animations of field lines to improve their students' insight into the dynamic effect of electromagnetic fields. Dori and Belcher [32] used these animations as technology-based learning materials, and studied the effect on students' conceptual understanding. They reported that students who used these materials scored significantly higher on post-test questions. Similar findings were communicated by Sousa et al. [33].

Törnkvist et al. [34] explained that many university students have difficulties assigning the correct characteristics to field lines. For example, about $85 \%$ of their second-year electromagnetism students did not notice that field lines can never cross. Others incorrectly stated that field lines cannot form loops, or claimed that field lines should always be closed. Törnkvist et al. also found some indications of difficulties with switching between field vectors and field lines when interviewing students. Therefore, they acknowledged that the differences between both representations should be discussed during instruction.

Gire and Price [35] reported on a variety of errors students make when sketching graphical representations of vector fields, and argued that instructors should be aware that some representational features may have two potential meanings (e.g., length, meaning both distance between points and strength of a field) or do not match what is being represented (e.g., closer spacing in field line diagrams corresponds to a greater magnitude). In addition, Fredlund et al. [36] stated that some representations are being rationalized (e.g., represent a complex object as a dot in a diagram), meaning certain aspects of a representation are taken for granted, while students may not understand all the properties or characteristics. Therefore, it is important to unpack these representations: we have to "show" students the intended meaning of all the parts that should not be considered obvious in the representation.

In our work on the use of vector calculus in electrodynamics [37-40], we observed that students struggle with graphical representations of vector fields when they were asked to interpret divergence and curl of electromagnetic fields. During semistructured individual interviews [38], many students opted to draw field line diagrams when they were asked to solve problems involving electromagnetic fields, but failed to do so properly. In addition, many students described divergence as a quantity related to the spreading of field lines, and curl as a measure of how much field lines bend. However, we could not determine if these problems were caused by conceptual difficulties regarding the vector operators, with a failure to construct or interpret visualizations of vector fields, or both. In this study, we investigate how students' representational fluency with field vector plots and field line diagrams may have had influenced previous results.

Considering our own experiences and the results from the literature, we have built our study on three research questions:

(1) What difficulties do students encounter with interpreting and sketching field line diagrams and field vector plots? 
(2) What difficulties do students encounter when switching between symbolic and graphical representations of vector fields?

(3) To what extent are the identified difficulties regarding the use of various representations of vector fields generalizable?

The research reported on in this paper comprises a qualitative and a quantitative part and was conducted at four universities: KU Leuven (Belgium), University of Hull (UK), University of St Andrews (UK), and Dublin City University (Ireland). In Sec. II we provide some information about the educational context and prior knowledge of the various student cohorts. We describe how we used qualitative data from student interviews and field notes during tutorial sessions to design a written paper-and-pencil questionnaire, called the Vector Field Representations (VFR) test. We also describe the conditions in which students from all four universities took the VFR test and discuss the analysis procedure. In Sec. III, we present a detailed overview of our results. Multiple examples of student responses to the VFR test questions are shown, and we explain how prevalent the identified difficulties are. The key results are discussed in Sec. IV, where we also comment on implications for instruction. In Sec. V, we summarize the outcomes of our study and propose further lines of investigation.

\section{RESEARCH DESIGN}

In Sec. II A, we discuss the educational context at all four universities that are involved in this research project. While we do not aim to compare universities or courses, understanding differences in prior knowledge is important when interpreting the results in Sec. III. In the first part of Sec. II B, we discuss the methodology of the qualitative studies that were conducted in this research project. In the second part we explain how those results were adopted to design the VFR test, and how responses to this questionnaire were analyzed in order to answer our research questions.

\section{A. Educational contexts}

At KU Leuven, three semistructured student interviews were conducted in the autumn of 2015 with students who finished a second-year electrodynamics course. At the start of the academic year in October 2016, the second-year physics and mathematics majors enrolled in an intermediate mechanics course took the VFR test $(N=36)$. They had already successfully completed an introductory course on electromagnetism, using Giancoli's textbook [41], and a calculus course including multiple chapters on vector fields and vector calculus, based on the book by Adams and Essex [42]. As graphical representations of electromagnetic fields are treated both in the physics curriculum at secondary school and the introductory electricity and magnetism course at university, we expected the students to be able to interpret and construct field line diagrams and field vector plots. In addition, they would have had extensive practice in switching between symbolic and graphical representations during the problem-solving sessions of the calculus course.

The students at the University of Hull who took the VFR test $(N=57)$ were all enrolled in either a BSc or integrated masters program in the School of Mathematics and Physical Sciences. All of these students had received instruction in the previous semester on the use of graphical and symbolic representations of electric and magnetic fields, in an introductory electromagnetism course using the textbooks by Halliday, Resnick, and Walker [43], and Duffin [44].

The University of St. Andrews students that participated in our study $(N=50)$ were enrolled in a mathematical methods course for students in the School of Physics and Astronomy, and were in either their second or third year. All of them were on BSc or integrated masters programs, studying experimental or theoretical physics, some of them jointly with mathematics or chemistry. They had all taken introductory electromagnetism during the previous semester, which was taught using the textbook by Halliday, Resnick, and Walker [43]. 40\% of them had also taken a course on vector calculus in the previous semester that was taught by the School of Mathematics and Statistics using the textbook by Adams and Essex [42]. The introductory electromagnetism module emphasized conceptual understanding and the use and interpretation of vector field plots and line diagrams, whereas the vector calculus course placed greater emphasis on computation skills.

At Dublin City University (DCU), students enrolled in physics, electronic engineering, and preservice science teacher courses $(N=53)$ took the VFR test at the start of a second-year, second-semester electromagnetism module in 2017. In the same course, field notes were taken during the tutorial sessions in 2016. All students of both cohorts had taken an introductory physics course; some of them a calculus-based physics course that used Young and Freedman's textbook [45], others the algebra-based version of Giancoli's textbook [41]. They had not taken any mathematics courses that would have emphasized graphical representations of vectors fields, but would typically have encountered electric field representations in secondary and introductory university physics modules.

\section{B. Methodology \\ 1. Qualitative data collection procedure}

Since we observed that some of our students struggled with graphical representations of vector fields when they were asked to interpret the divergence and curl of electromagnetic fields $[37,38]$, we followed up with three individual semi-structured student interviews at KU Leuven where we asked the volunteers to switch between field line diagrams, field vector plots, and algebraic expressions of vector fields while thinking aloud. Despite having taken the 
relevant prior courses, all three participants exhibited several difficulties when constructing visualizations of vector fields, or needed help from the interviewer to solve the tasks. The identified difficulties, which are discussed in the next section, were listed and categorized in terms of representation (field lines, field vectors, or symbolic expression) and characteristic of the field (magnitude, direction, or other).

During the spring of 2016, we gave similar tasks to physics and engineering students at DCU who were enrolled in the aforementioned second-year electromagnetism course. These worksheets were part of the course material and were solved by groups of students during several 50-min tutorial sessions. Listening to student discussions, keeping track of their activities, and making extensive field notes about the problems that they encountered allowed us to make the list of student difficulties more accurate.

\section{Design and analysis of the VFR test}

In order to further refine the list of common student difficulties and determine their prevalence, we designed a written paper-and-pencil test that consists of open-ended questions that were asked during the interviews at $\mathrm{KU}$ Leuven and problem-solving sessions at DCU. This questionnaire, called the Vector Field Representations test, is presented in Fig. 1. It asks students to interpret and construct both symbolic and graphical representations of vector fields, and to comment on some statements that correspond to common student difficulties. The links between the questions and the different representations are presented schematically in Fig. 2.

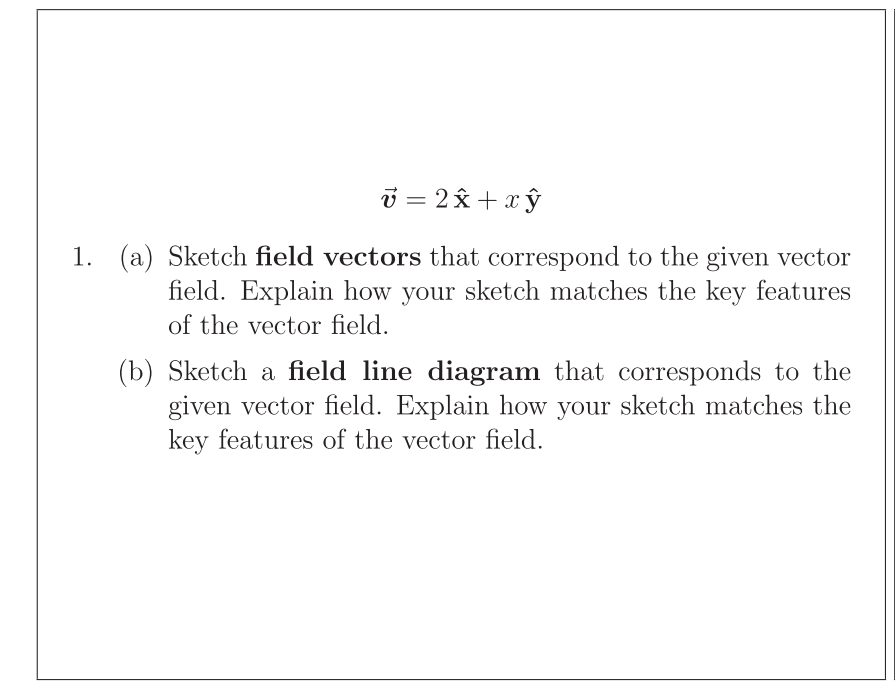

(a) Question 1

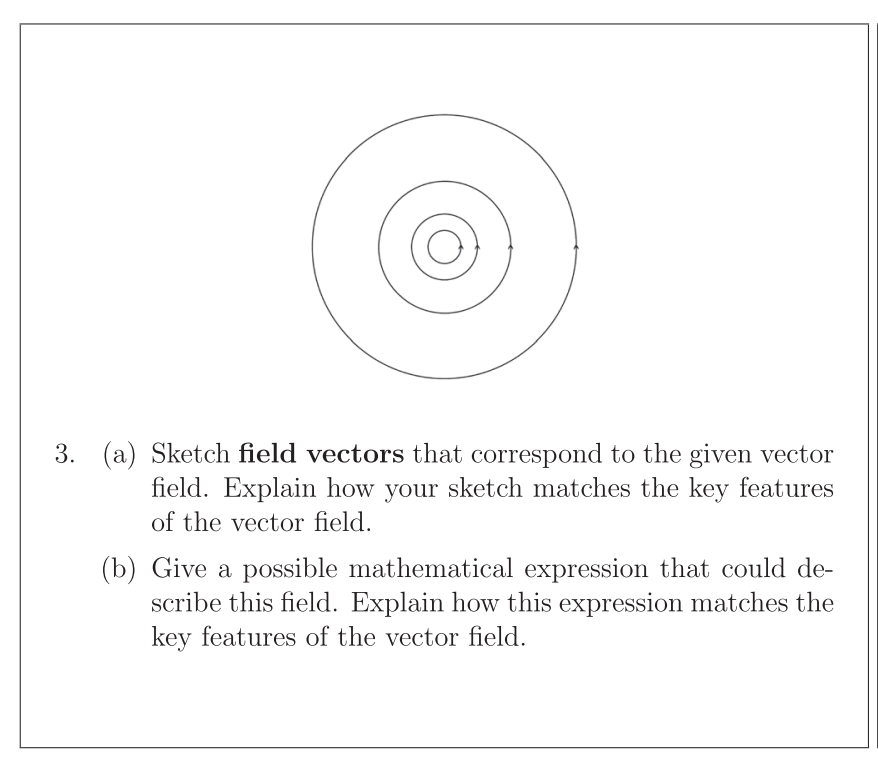

(c) Question 3

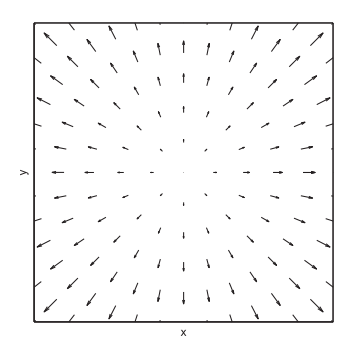

2. (a) Sketch a field line diagram that corresponds to the given vector field. Explain how your sketch matches the key features of the vector field.

(b) Give a possible mathematical expression that could describe this field. Explain how this expression matches the key features of the vector field.
4. Comment on the following statements. Explain in detail why you agree or disagree.

(a) Field lines can touch each other or cross.

(b) Field vectors can touch each other or cross.

(c) The magnitude of the field is zero at point a.

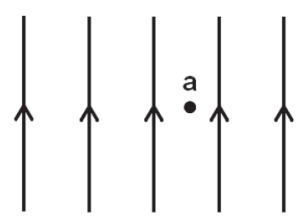

(d) In some cases, a field line may merge with another field line.

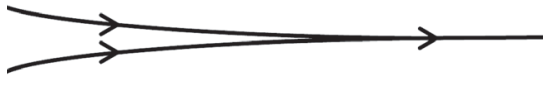

(d) Question 4

FIG. 1. Questions on the VFR test. 


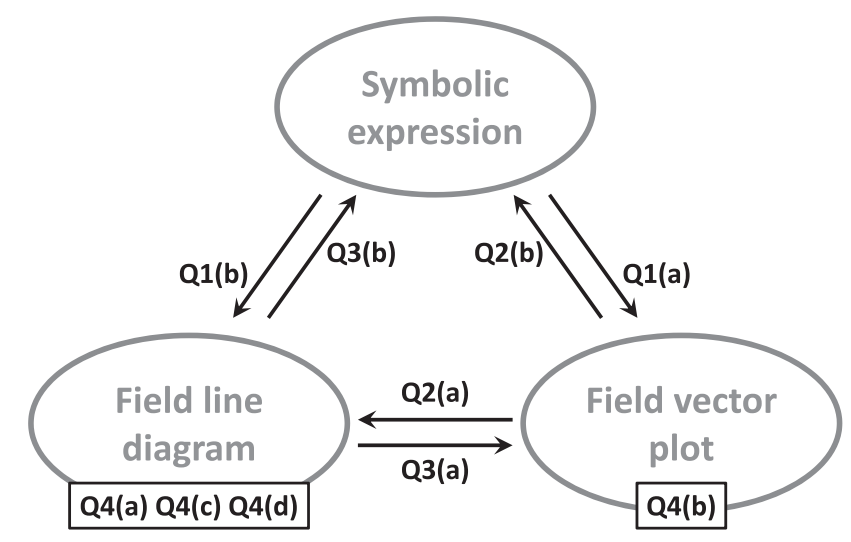

FIG. 2. Overview of the relations between the representations of vector fields and the questions on the VFR test.

The questions on the VFR test are in accordance with the curriculum at all four universities, and were already discussed during student interviews and problem-solving sessions. During these qualitative studies, we asked students to think aloud and made sure that they interpreted the questions as intended. In addition, we asked whether they had learned about field vector plots and field line diagrams. As all students agreed they had come across such visualizations before, the test has a high degree of validity. In addition, the questionnaire was informally discussed with instructors at different institutions, who agreed that it was a valid test to study representations of vector fields.

The VFR test was taken by students of KU Leuven, University of Hull, St Andrews, and DCU $(N=196)$ under examlike conditions, meaning that students worked individually, were not allowed to communicate with each other, and had no textbooks or notes at their disposal. While the test was not formally graded, students were asked to answer to the best of their knowledge and explain their responses in detail. The vector notation in the questions was adapted to match the notation style that was most commonly used by the students, depending on the university and course textbook. All students filled in an informed consent form, and finished the test in about 30-40 min.

The responses to the VFR questions were initially analyzed based on the list of difficulties that emerged from student interviews conducted at KU Leuven, and field notes made during in-class activities at DCU. The first author of this paper then designed a coding scheme, extended the list of difficulties based on the responses to the VFR test, and coded all responses using this coding scheme. The final version of the categorized list of identified student difficulties is discussed in Sec. III. The co-authors of the paper then applied the same coding to the responses, so that two researchers coded each response. To verify the presented categorization, we evaluated the interrater reliability by calculating Cohen's kappa $(\kappa)$ [46]. For most of the categories in individual questions, Cohen's kappa was at least 0.63 , indicating a substantial to almost perfect agreement. For a few categories kappa was lower than 0.63 , but since the percentage agreement was at least 90\% in these cases, the low kappa score can be attributed to the fact that such difficulties were rather rare. Therefore, a low kappa does not necessarily reflect a low rate of agreement [47].

In the next section, we will show that similar difficulties were experienced by students from different universities. While the prevalence of the observed errors may depend on the institution, being able to make a categorization scheme that works for all four student cohorts is an indication that the test has a high reliability.

\section{RESULTS}

The first goal of our study was to determine the kinds of difficulties students typically encounter when interpreting and constructing field vector plots, field line diagrams, and symbolic expressions of vector fields. As mentioned above, a list of student difficulties related to these representations was established after the student interviews, observations of in-class activities, and an initial analysis of responses on the VFR test. The most common errors were subdivided into groups of difficulties related to the magnitude of the vector field, related to the direction of the vector field, and other difficulties. Table I shows the final categorization. As all researchers found this categorization useful and applicable to all questions on the VFR test, it serves as the answer to our first research question. While we do not have sufficient proof to generalize to other student populations, we feel that it can act as an indication of the kinds of difficulties that can be expected when students use vector field representations.

To answer the remaining two research questions, we present and discuss some of the most prevalent responses to the questions on the VFR test, and supplement these data with observations that were made during the individual interviews and in-class discussions. In addition, we report on the prevalence of the identified student difficulties using the abbreviations found in the third column of Table I. In Sec. IV, we propose some possible causes and solutions.

This section contains three subsections. We discuss the prevalence of difficulties with switching from algebraic expressions or field line diagrams to field vector plots (Sec. III A), with constructing field line diagrams (Sec. III B), and with setting up symbolic expressions of vector fields (Sec. III C). In some cases, the relative prevalence of the difficulties varies between student populations, due to differences in educational context. Because we do not aim to compare universities, we did not perform an extended statistical analysis; the results merely indicate that most difficulties can be found in four different European universities. This allows the reader to focus on the identified student difficulties, and not be distracted by institutional differences. 
TABLE I. Final categorization of student difficulties with vector representations. The first four columns indicate how the difficulties were categorized in terms of representations and characteristics of the field. The numbers in the last column correspond to the questions on the VFR test (Fig. 1).

\begin{tabular}{|c|c|c|c|c|}
\hline Representation & Characteristic & Error & Description & Question(s) \\
\hline \multirow[t]{9}{*}{ Field vectors } & \multirow[t]{3}{*}{ Magnitude } & VM1 & Arrow length does not match magnitude of the field & $1 \mathrm{a}, 3 \mathrm{a}$ \\
\hline & & VM2 & Only one set of vectors is drawn & $1 \mathrm{a}, 3 \mathrm{a}$ \\
\hline & & $\mathrm{VM}^{\mathrm{a}}$ & Density represents magnitude as well & $3 \mathrm{a}$ \\
\hline & \multirow[t]{2}{*}{ Direction } & VD1 & Difficulties related to addition of vectors & 1a \\
\hline & & VD2 & Field vectors are not tangent (e.g., perpendicular) to field lines & $3 \mathrm{a}$ \\
\hline & \multirow[t]{4}{*}{ Other } & VO1 & Arrows are centered on their location & $1 \mathrm{a}, 3 \mathrm{a}$ \\
\hline & & VO2 & All vectors start at the origin & $1 \mathrm{a}$ \\
\hline & & VO3 & Sketched vectors are bent instead of straight arrows & $3 a$ \\
\hline & & VO4 & Vectors are not allowed to touch or cross & $4 \mathrm{~b}$ \\
\hline \multirow[t]{7}{*}{ Field lines } & Magnitude & LM1 & Density does not represent magnitude correctly & $1 \mathrm{~b}, 2 \mathrm{a}$ \\
\hline & \multirow[t]{2}{*}{ Direction } & $\mathrm{LD}^{\mathrm{b}}$ & Direction of the field is not indicated (no arrows) & $1 \mathrm{~b}, 2 \mathrm{a}$ \\
\hline & & LD2 & Field lines are not tangent (e.g., perpendicular) to field vectors & $1 \mathrm{~b}, 2 \mathrm{a}$ \\
\hline & \multirow[t]{4}{*}{ Other } & LO1 & Lines do not start or end at correct locations & $1 \mathrm{~b}, 2 \mathrm{a}$ \\
\hline & & $\mathrm{LO} 2$ & Field is zero in between lines & $4 \mathrm{c}$ \\
\hline & & LO3 & Lines are allowed to split or merge & $4 d$ \\
\hline & & $\mathrm{LO} 4$ & Lines are allowed to touch or cross & $4 a, 4 d$ \\
\hline \multirow[t]{3}{*}{ Symbolic } & Magnitude & SM1 & Expression does not match magnitude of the field & $2 b, 3 b$ \\
\hline & \multirow[t]{2}{*}{ Direction } & SD1 & Expression does not match the direction of the field & $2 b, 3 b$ \\
\hline & & SD2 & No use of (unit) vectors & $2 b, 3 b$ \\
\hline
\end{tabular}

${ }^{\mathrm{a}}$ This is not necessarily incorrect, but might indicate some confusion with field lines.

${ }^{b}$ While this is not incorrect, this indicates students do not take full advantage of the representation.

Certain difficulties, for example, with the depiction of the magnitude of the vector field by the arrow length (VM1), can occur in every sketch of a field vector diagram, irrespective of the original representation (field line diagram or symbolic expression). However, as will be discussed later, this difficulty may have different causes, and possibly depends on which representation was used as the starting point. Therefore, the subsections are subdivided in accordance with the structure of the VFR test (Fig. 1 and 2). This also helps to answer the second research question more precisely.

\section{A. Student difficulties related to sketching field vector plots}

1. Difficulties with switching from a symbolic expression to a field vector plot. While administering the tutorials at DCU, we observed that many students struggle with switching from symbolic to graphical representations of vector fields. When constructing a field vector plot, they typically failed to visualize how the magnitude and direction of the vector field change with distance from the origin, because they incorrectly added the $x$ and $y$ components of a vector field at representative locations in the field.

Question 1(a) of the VFR test [Fig. 1(a)] aims to further investigate such difficulties: students were asked to sketch a field vector plot that corresponds to the algebraic expression $\mathbf{v}=2 \hat{\mathbf{x}}+x \hat{\mathbf{y}}$. Some typical responses to this assignment are shown in Fig. 3. To discuss to what extent the identified difficulties are prevalent, we summarize the results from each cohort in Table II. Incorrect responses are labeled using the abbreviations from Table I. Since a student can make multiple errors in one sketch, the percentages do not sum to 100 . Students who gave an incoherent or incomprehensible answer are categorized as "No answer." It is clear from Table II that many difficulties occur in different universities, albeit with different prevalences.

An example of an entirely correct student sketch is presented in Fig. 3(a). Some students drew arrows centered on their location (VO1), similar to Fig. 3(b), often with an excellent explanation of how the field vector plot should be constructed. Such responses were only observed at one of the universities, and there is no clear indication of the cause of this difficulty.

The student who drew the response shown in Fig. 3(c) also gave a correct explanation:

"The field is constant in the $x$ direction, and varies with $x$ in the $y$ direction."

Nevertheless, the response reveals difficulties with drawing the direction (VD1) and magnitude (VM1) of the vector field. Corresponding explanations to similar responses imply that this error was often caused by incorrectly adding vectors. 


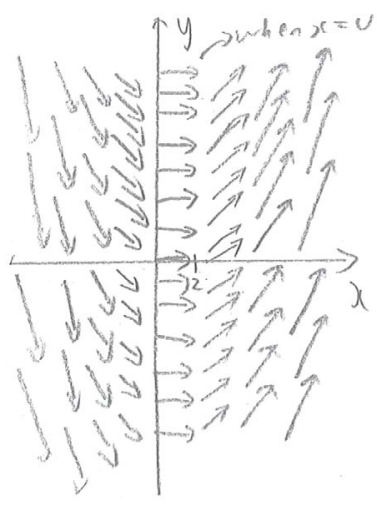

(a) Correct.

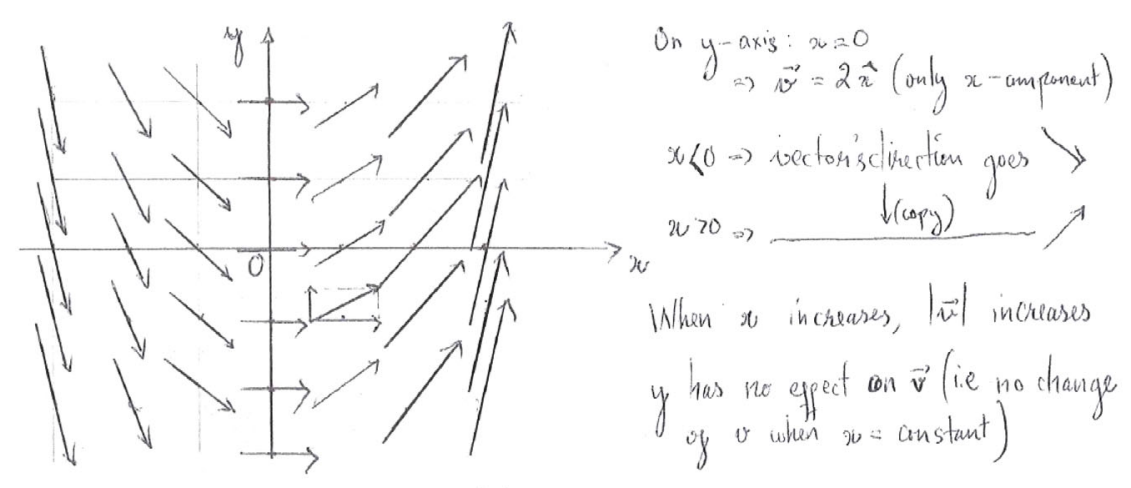

(b) Error VO1.

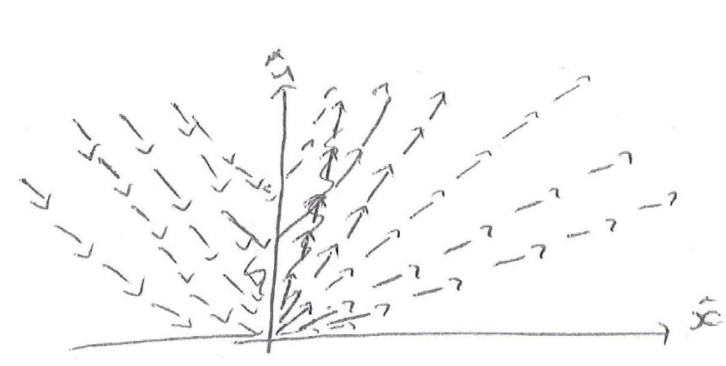

(c) Errors VM1 and VD1.

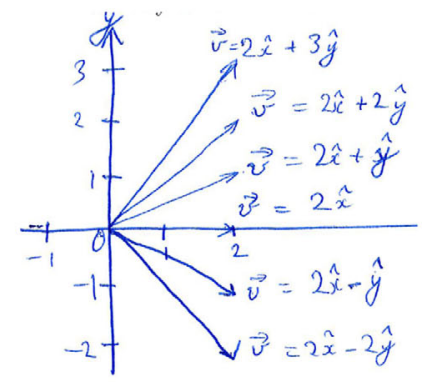

(d) Errors VM2, VD1, and VO2.

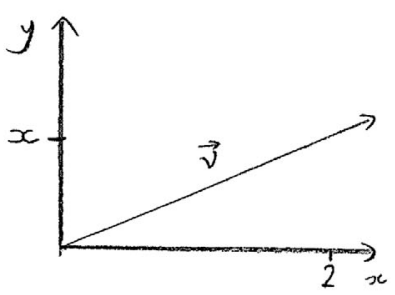

(e) Error VM2, VD1, and VO2.

FIG. 3. Examples of student sketches as a response to question 1(a) [Fig. 1(a)].

The last two examples of student responses demonstrate that many students may not have known how to sketch a field vector plot at all, or may not know what a vector field is. While such responses were rarely seen at some universities, they were very prevalent at others (up to 60\%). Figure 3(d) shows a field vector plot with no indication of how the magnitude of the field changes, since only a single set of vectors is drawn (VM2) which all start at the origin (VO2). Notice that responses that were coded as VM2 were almost never coded as VM1, since we lacked information about how the magnitude of the field changes when the

TABLE II. Summary of responses to question 1(a) [Fig. 1(a)]. Incorrect responses are associated with the errors listed in Table I. Since students can exhibit multiple difficulties in a single sketch, the percentages do not sum to 100 .

\begin{tabular}{ccccc}
\hline \hline & $\begin{array}{c}\text { KUL } \\
(n=36)\end{array}$ & $\begin{array}{c}\text { UoH } \\
(n=57)\end{array}$ & $\begin{array}{c}\text { St A } \\
(n=50)\end{array}$ & $\begin{array}{c}\text { DCU } \\
(n=53)\end{array}$ \\
\hline Correct & $56 \%(20)$ & $0 \%(0)$ & $26 \%(13)$ & $2 \%(1)$ \\
Incorrect & $42 \%(15)$ & $70 \%(40)$ & $74 \%(32)$ & $91 \%(48)$ \\
VM1 & $27 \%$ & $18 \%$ & $41 \%$ & $10 \%$ \\
VM2 & $53 \%$ & $83 \%$ & $14 \%$ & $73 \%$ \\
VD1 & $80 \%$ & $93 \%$ & $65 \%$ & $83 \%$ \\
VO1 & $0 \%$ & $0 \%$ & $38 \%$ & $0 \%$ \\
VO2 & $40 \%$ & $33 \%$ & $3 \%$ & $54 \%$ \\
Other & $13 \%$ & $20 \%$ & $0 \%$ & $13 \%$ \\
No answer & $3 \%(1)$ & $30 \%(17)$ & $0 \%(0)$ & $8 \%(4)$ \\
\hline \hline
\end{tabular}

sketch did not show field vectors at increasing distances. The multivalued nature of the sketched field in Fig. 3(d) indicates difficulties with vector addition and decomposition (VD1): the students seem to confuse vector field components with coordinates. In more extreme cases, students would even only draw a single vector, as in Fig. 3(e). In this example, the student did not assign a numerical value for $x$ on the $y$ axis, and also seems to struggle with vector addition (VD1). However, we cannot exclude that other factors, like rote learning or misunderstanding what a vector field is, are in play as well.

A minority of students sketched bent arrows or confused field vectors and field lines. Since this was only seen in a few answers, such errors were categorized as "Other." In general, the examples in Fig. 3 and the results in Table II indicate that most errors were related to difficulties with adding vectors, resulting in arrows with an incorrect direction (VD1) and magnitude of the field (VM1). Since difficulties with vector addition were seen so often in this assignment, they are discussed in more detail in Sect. IV.

2. Difficulties with switching from a field line diagram to a field vector plot.

In question 3(a) [Fig. 1(c)], students were asked to draw a field vector plot that corresponds to a given field line diagram. Typical responses are shown in Fig 4, and the prevalence of the difficulties observed in responses to question 3(a) can be found in Table III. 


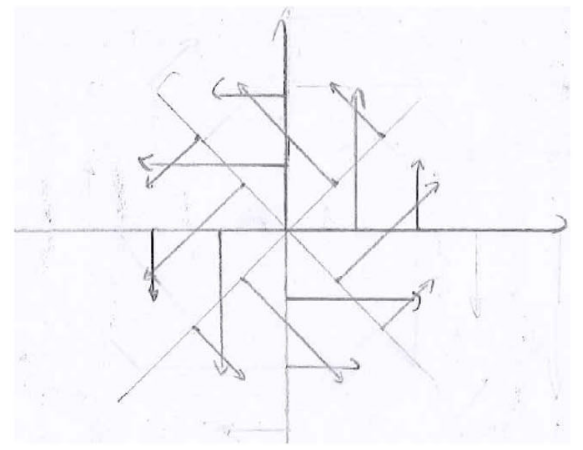

(a) Correct.

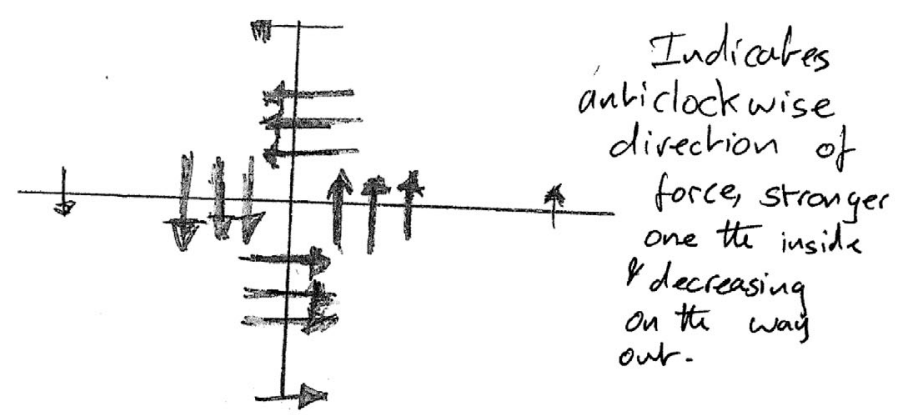

(b) Errors VM3 and VO1.

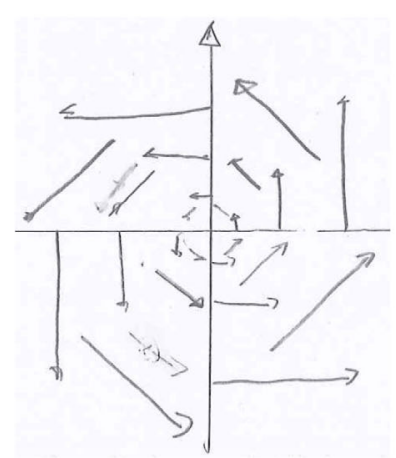

(c) Error VM1.

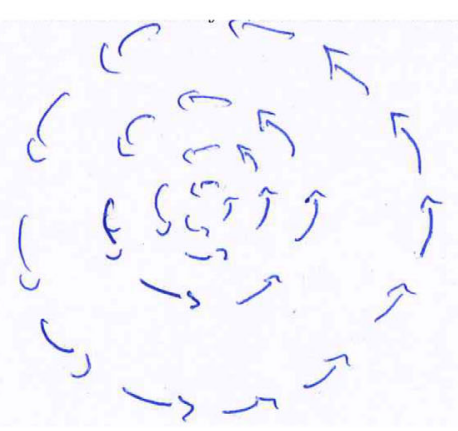

(d) Errors VM1, VM3, and VO3.

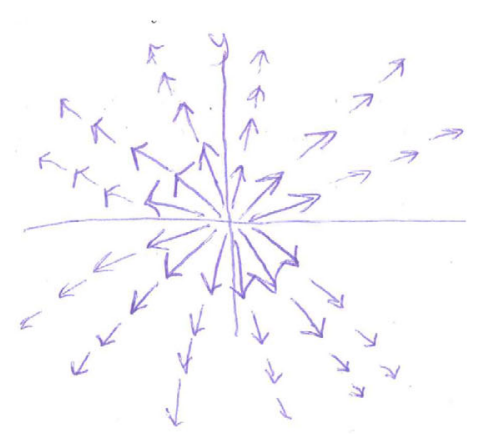

(e) Error VD2.

FIG. 4. Examples of student sketches as a response to question 3(a) [Fig. 1(c)].

An example of a correct sketch is shown in Fig. 4(a). Notice that this student let the vector arrows cross, which is permitted since vector arrows only carry information about the point where they originate from, and, therefore, explicitly shows he does not exhibit difficulty VO4.

The sketch and corresponding explanation in Fig. 4(b) indicate that this student understood how the magnitude of the field behaves. However, the arrows are centered on their location (VO1) as they do not start on the $x$ and $y$ axis while being vertical or horizontal, respectively. Similar

TABLE III. Summary of responses to question 3(a) [Fig. 1(c)]. Incorrect responses are associated with the errors listed in Table I. Since students can exhibit multiple difficulties in a single sketch, the percentages do not sum to 100 .

\begin{tabular}{ccccc}
\hline \hline & $\begin{array}{c}\text { KUL } \\
(n=36)\end{array}$ & $\begin{array}{c}\mathrm{UoH} \\
(n=57)\end{array}$ & $\begin{array}{c}\text { St A } \\
(n=50)\end{array}$ & $\begin{array}{c}\text { DCU } \\
(n=53)\end{array}$ \\
\hline Correct & $11 \%(4)$ & $2 \%(1)$ & $12 \%(6)$ & $0 \%(0)$ \\
Incorrect & $86 \%(31)$ & $68 \%(39)$ & $88 \%(44)$ & $38 \%(20)$ \\
VM1 & $90 \%$ & $46 \%$ & $48 \%$ & $85 \%$ \\
VM2 & $3 \%$ & $18 \%$ & $0 \%$ & $10 \%$ \\
VM3 & $16 \%$ & $8 \%$ & $5 \%$ & $0 \%$ \\
VD2 & $10 \%$ & $74 \%$ & $14 \%$ & $40 \%$ \\
VO1 & $39 \%$ & $5 \%$ & $68 \%$ & $20 \%$ \\
VO3 & $19 \%$ & $8 \%$ & $11 \%$ & $30 \%$ \\
Other & $3 \%$ & $21 \%$ & $0 \%$ & $0 \%$ \\
No answer & $3 \%(1)$ & $30 \%(17)$ & $0 \%(0)$ & $62 \%(33)$ \\
\hline \hline
\end{tabular}

difficulties were also observed in the previous question 1(a). In the particular case of Fig. 4(b), the field vectors are further away from each other when the field strength decreases, matching the density of the field lines (VM3). While this is not incorrect, it may indicate that students are confused about what the density of field lines represents, or that they think that the density of field vectors has a meaning. Some students exhibited similar difficulties during the tutorials at DCU, but failed to state what kind of field property is represented by the density of field vectors. These observations imply that some students may confuse characteristics of field lines and field vectors, and demonstrate a poor fluency with both visualizations.

The diagram in Fig. 4(c) shows the most prevalent difficulty with question 3(a): the length of the arrow does not represent the magnitude of the field correctly (VM1). While the same difficulty was seen in question 1(a), the cause of this error seems to be related to the format of the original representation. From observations made during in-class activities, follow-up questions during individual student interviews, and the findings that are described in the next sections, we suspect that students misunderstood how the field line density represents the field strength in question 3(a), while errors in question 1(a) were often caused by incorrectly adding vectors. Both difficulties are discussed in Sec. IV.

Figure 4(d) is another example of an incorrect representation of the magnitude of the field (VM1), as the 
lengths of the arrows should decrease with increasing distance from the center. Again, the density of the arrows seems to match the density of the field lines (VM3). On top of these difficulties, the sketched vectors in Fig. 4(d) are also bent or curved (VO3). This may be a composite error of thinking a charge particle would follow a field line, and wishing to represent this by showing that "therefore" the force is also following a curve path.

Many students seemed to confuse the characteristics of field lines and equipotential lines. Consequently, they misinterpreted the given field line diagram, and produced a sketch similar to the one in Fig. 4(e), in which field vectors are perpendicular to field lines (VD2). In some instances, students explicitly stated that they had been given an illustration of equipotential lines. However, students may also have sketched such a figure due to rote memorization of a radial field vector plot, as this is a common example in both physics and mathematics. Nevertheless, this student correctly determined how the magnitude of the field changes:

\section{"Center has strongest magnitude (field lines closest) $\rightarrow$ decreases as $r$ increases."}

The majority of the student responses to question 3(a) were similar to the sketches in Fig 4. A few students sketched only a single vector or seemed to confuse field lines with field vectors, and their responses were therefore categorized as Other.

3. Other difficulties with field vector plots.

Questions 1(a) and 3(a) did not allow us to determine to what extent students (incorrectly) believe that vectors are not allowed to cross (VO4), as they can simply avoid this problem by choosing an appropriate scale for the length of the vectors. Because all three participants of the student interviews at KU Leuven struggled with this statement, we explicitly asked in question 4(b) [Fig. 1(d)] whether vectors are allowed to touch or cross.

Figure 5 shows that less than $50 \%$ of the students agree with this statement, and an even smaller proportion gave a

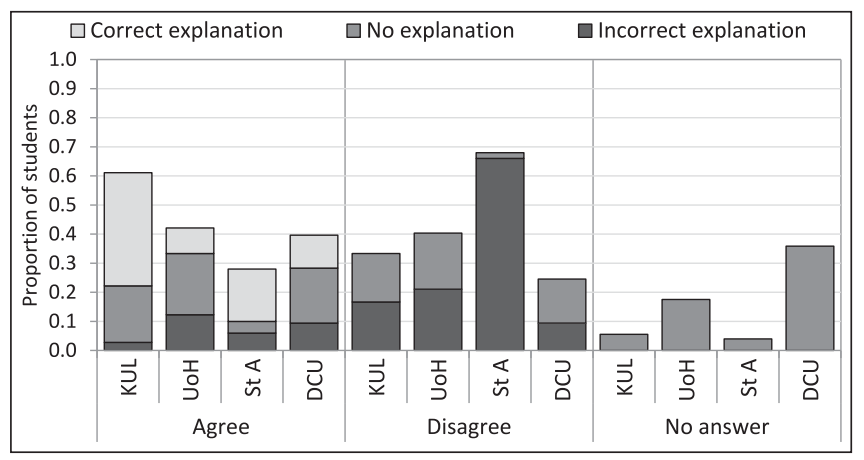

FIG. 5. Distribution of responses and corresponding argumentation to question 4(b) [Fig. 1(d)]. valid argument. Some students produced excellent explanations to argue why the statement is correct:

"Field vectors can touch each other or cross, but we cannot have two different field vectors originating at the same point because the function would be multivalued." "Correct. The length of a vector represents the magnitude of the field, but the scale is an arbitrary choice. Therefore, it is possible to choose a scale for which vectors cross."

However, some students agreed with the statement with incorrect reasoning:

"Agree, we can combine vectors to give a resultant vector."

This student may have imagined a vector field with multiple values or directions at a single point, or may have been thinking about adding vectors by placing them "tip-to-tail" to find the resultant vector. When analyzing the responses of students who disagreed with the statement, it seemed like many students confused characteristics of field lines and field vectors, for example,

"I disagree with the statement because crossing vectors would signify that the field is moving in multiple directions."

This may indicate students struggle with the idea that a field vector arrow only gives information about the point where it originates from (its "tail"). It is also possible that the students confused characteristics of field vectors and field lines.

\section{B. Student difficulties related to sketching field line diagrams}

1. Difficulties with switching from a symbolic expression to a field line diagram.

During the interviews at KU Leuven, we found that when students are asked to sketch a given field, they often draw field line diagrams instead of field vector plots. However, they seemed to lack the required representational fluency to produce a diagram that contains all necessary characteristics of the field. Question 1(b) aims to document student difficulties with sketching a field line diagram, starting from a symbolic expression. Nevertheless, it is likely that students also based their answer (partly) on the field vector plot that was sketched as response to question 1(a). The prevalences of the difficulties described below are presented in Table IV.

The given field increases with distance from the $y$ axis, but has zero divergence, meaning field lines should not start or end anywhere in space. Fig. 6(a) is an example of a correct answer, including a correct explanation. Since it is a 
TABLE IV. Summary of responses to question 1(b) [Fig. 1(a)]. Incorrect responses are associated with the errors listed in Table I. Since students can exhibit multiple difficulties in a single sketch, the percentages do not sum to 100 .

\begin{tabular}{ccccc}
\hline \hline & $\begin{array}{c}\text { KUL } \\
(n=36)\end{array}$ & $\begin{array}{c}\mathrm{UoH} \\
(n=57)\end{array}$ & $\begin{array}{c}\text { St A } \\
(n=50)\end{array}$ & $\begin{array}{c}\text { DCU } \\
(n=53)\end{array}$ \\
\hline Correct & $56 \%(20)$ & $0 \%(0)$ & $46 \%(23)$ & $4 \%(2)$ \\
LD1 & $40 \%$ & $\cdots$ & $4 \%$ & $0 \%$ \\
Incorrect & $39 \%(14)$ & $47 \%(27)$ & $44 \%(22)$ & $40 \%(21)$ \\
LM1 & $71 \%$ & $100 \%$ & $68 \%$ & $67 \%$ \\
LD1 & $43 \%$ & $41 \%$ & $23 \%$ & $33 \%$ \\
LD2 & $14 \%$ & $63 \%$ & $36 \%$ & $48 \%$ \\
LO1 & $43 \%$ & $44 \%$ & $59 \%$ & $33 \%$ \\
Other & $7 \%$ & $4 \%$ & $27 \%$ & $33 \%$ \\
No answer & $6 \%(2)$ & $53 \%(30)$ & $10 \%(5)$ & $57 \%(30)$ \\
\hline \hline
\end{tabular}

difficult task to correctly construct a field line diagram in such a way that the field lines get denser as $x$ increases, we counted all similar drawings as correct unless it was explicitly stated that the density of the field lines did not change or should decrease with increasing $x$ values. This means we may have somewhat underestimated the fraction of students who have difficulty relating the density of the field lines to the strength of the field (LM1).

A typical incorrect sketch is shown in Fig. 6(b). In this diagram, field lines emerge from the $y$ axis (LO1). As this is a divergence-free field, there should be no location where field lines start or end. Other students produced similar sketches where field lines started at the $x$ axis.

The majority of students constructed a diagram with field lines that are tangent to the field vectors they sketched as a

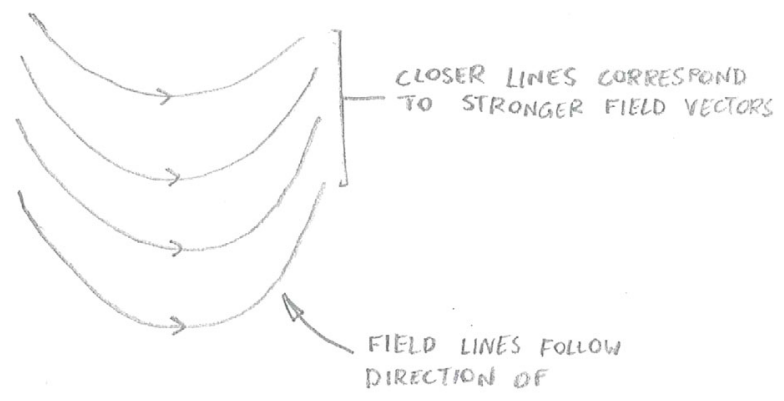

(a) Correct.

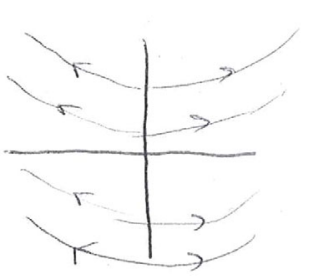

(b) Error LO1.

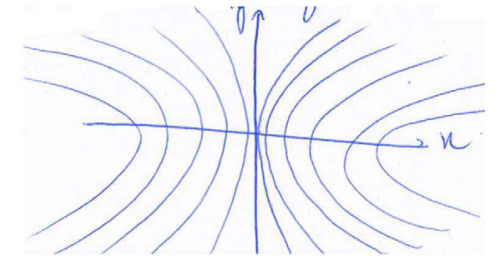

(c) Errors LM1, LD1, and LD2.
FIG. 6. Examples of student sketches as a response to question 1(b) [Fig. 1(a)]. response to question 1(a). However, some students constructed a decent field vector plot as a response to question 1(a), but drew field lines that did not correspond to the field vector plot in their previous answer. A student who gave a correct answer to question 1(a) similar to Fig. 3(a) produced the sketch in Fig. 6(c). In this diagram, the field lines are not tangent to the arrows (LD2), do not indicate the direction of the field (LD1), and incorrectly represent the magnitude of the field (LM1).

Table IV shows that many students seemed unsure about what field lines are, and some even claimed that they did not encounter this in class. This resulted in a lot of blank answer sheets and incoherent responses. Nevertheless, field line diagrams were part of the curriculum and textbooks used in these students' prior courses, as discussed in Sec. II A. Therefore, we think the reason that students do not remember field line diagrams is a lack of detailed discussions in class, rather than not mentioning it at all.

The most common difficulty is related to the magnitude of the field: a majority of students failed to represent the field line density correctly (LM1). Such errors may also have caused students to produce incorrect field vector plots in question 3(a). Difficulties related to the density of field lines as a measure for the magnitude of the field are extensively discussed in Sec. IV.

2. Difficulties with switching from a field vector plot to a field line diagram.

In question 2(a) [Fig. 1(b)], students were asked to draw a field line diagram that corresponds to a given field vector plot. The vector field in the illustration points in the radial direction, and the magnitude grows with increasing distance from the center of the field. This means the field line density should increase outwards as well. This is only possible if field lines emerge from various points in space, as is required in a field line diagram of a field with a nonzero divergence everywhere. While it is hard, if not impossible, to sketch a perfect diagram for this assignment, we were hoping for responses like the one in Fig. 7(a), which contains all necessary characteristics. However, as shown in Table V, only a minority of students constructed such a diagram. Typically, students' sketches were similar to Fig. 7(b) or Fig. 7(c).

The majority of students constructed a field line diagram like the one in Fig. 7(b), which fails to show how the magnitude of the vector field increases with increasing distance from the center (LM1). In addition, the field lines all originate from the center of the field (LO1). Some students explicitly wrote that something was wrong with the density of the field lines, but did not acknowledge this could be solved by letting field lines start "everywhere." Possibly, students are confused because such examples are rarely discussed during instruction, as we will argue in Sec. IV. In addition, a rote learning factor may also be at play, as the field line diagram is a very common example in electromagnetism (electric field of a positive charge). 


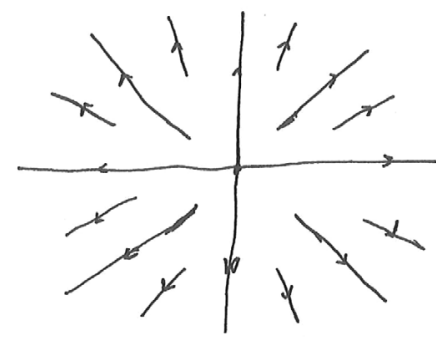

(a) Correct.

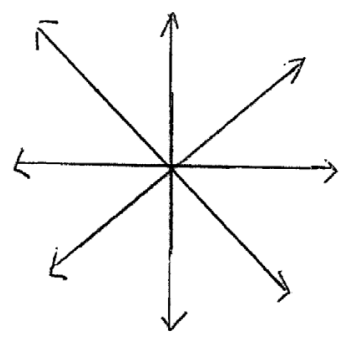

(b) Errors LO1 and LO3.

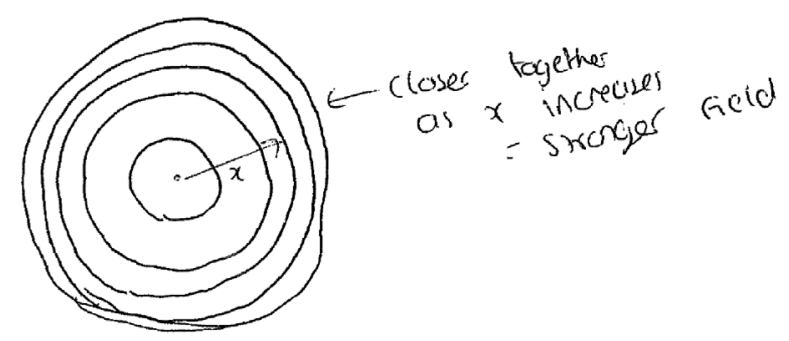

(c) Errors LD1 and LD2.

FIG. 7. Examples of student sketches as a response to question 2(a) [Fig. 1(b)].

Interestingly enough, we did not observe any student drawing splitting or merging field lines (LO3) to resolve the field line density issue, as seen during tutorial sessions at DCU.

In agreement with the findings from the previous sections, many students seemed to confuse characteristics of field lines and equipotential lines. These students produced sketches similar to Fig. 7(c), where field lines and field vectors are perpendicular (LD2) and often no direction was indicated (LD1). In this specific example, the student did acknowledge that the lines should be denser further from the center.

When comparing results from Table $\mathrm{V}$ to those of Table IV, it is interesting to see that students struggle with similar things when sketching a field line diagram, regardless of the symmetry of the field (Cartesian versus

TABLE V. Summary of responses to question 2(a) [Fig. 1(b)]. Incorrect responses are associated with the errors listed in Table I. Since students can exhibit multiple difficulties in a single sketch, the percentages do not sum to 100 .

\begin{tabular}{ccccc}
\hline \hline & $\begin{array}{c}\text { KUL } \\
(n=36)\end{array}$ & $\begin{array}{c}\text { UoH } \\
(n=57)\end{array}$ & $\begin{array}{c}\text { St A } \\
(n=50)\end{array}$ & $\begin{array}{c}\text { DCU } \\
(n=53)\end{array}$ \\
\hline Correct & $0 \%(0)$ & $0 \%(0)$ & $12 \%(6)$ & $0 \%(0)$ \\
Incorrect & $97 \%(35)$ & $89 \%(51)$ & $82 \%(41)$ & $43 \%(23)$ \\
LM1 & $97 \%$ & $78 \%$ & $88 \%$ & $100 \%$ \\
LD1 & $37 \%$ & $41 \%$ & $20 \%$ & $43 \%$ \\
LD2 & $23 \%$ & $67 \%$ & $29 \%$ & $39 \%$ \\
LO1 & $100 \%$ & $100 \%$ & $100 \%$ & $100 \%$ \\
Other & $7 \%$ & $0 \%$ & $0 \%$ & $0 \%$ \\
No answer & $3 \%(1)$ & $11 \%(6)$ & $6 \%(3)$ & $57 \%(30)$ \\
\hline \hline
\end{tabular}

cylindrical) and given representation (symbolic expression vs field vector plot). The most prevalent difficulties are related to an incorrect field line density (LM1) and incorrect starting points of the field lines (LO1). In addition, a considerable proportion of students seem to confuse characteristics of field lines and equipotential lines. Therefore, these three issues will be discussed in Sec. IV.

3. Other difficulties with field line diagrams.

In question 4(a) [Fig. 1(d)], we asked students whether field line diagrams are allowed to touch or cross (LO4). As can be seen from Fig. 8, the majority of students disagreed with this statement. Some students produced excellent arguments:

"Field lines cannot touch each other or cross because this would imply that the field was pointing in two different directions at the same time which cannot be true for a well defined vector field."

A small number of students agreed with the statement, but described a realistic situation that clarified their reasoning:

"I agree, because the field lines can start from the same point."

While this was not what was meant with the statement on the test, it was categorized as a correct answer.

Many students who acknowledged that field lines cannot touch or cross gave no explanation or stated that they knew this as a fact. During the individual student interviews, one of the interviewees also stated that he never really thought about a reason for field lines not to cross. Some students gave an incorrect reason for disagreeing with the statement, often related to confusing characteristics of field lines and equipotential lines:

"Disagree. Field lines connect areas of equal field strength, if they were to cross it would mean that the area would have 2 different field strengths simultaneously, which can't happen."

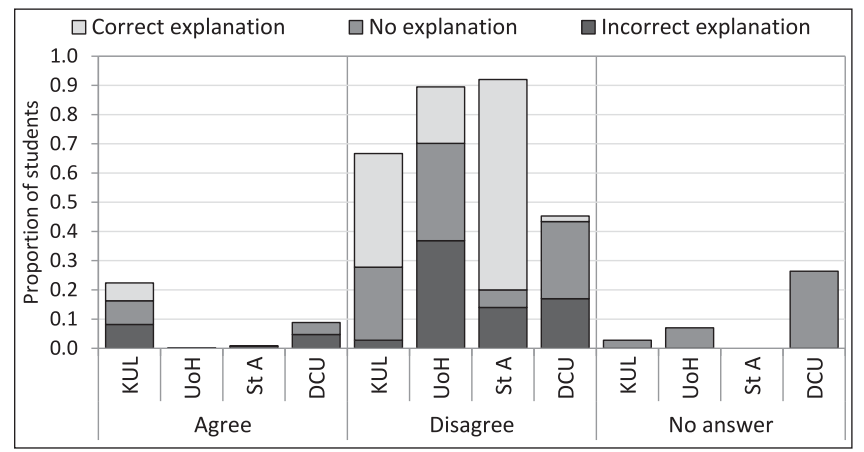

FIG. 8. Distribution of responses and corresponding argumentation to question 4(a) [Fig. 1(d)]. 
A few students believed that field lines are allowed to cross, and may have failed to consider the direction of the field at the location where field lines cross:

"Field lines can cross and touch each other because they do not show the magnitude of the field, only the direction."

In addition, this response indicates that the student did not take into account how the magnitude of a field is represented by the density of field lines.

When observing in-class student activities at DCU, we witnessed that some students have incorrect ideas about what happens in between field lines (LO2). The majority of students at all four universities disagreed with the statement that the field is zero in between field lines, as shown in Fig. 9. Most of them also gave a satisfying reason, for example,

"No, it is a uniform field and, therefore, the magnitude of the field is the same at each point, not zero."

Some students realize that the field is nonzero at every point, but give an incorrect explanation:

"No, the flux is zero, but that doesn't mean the field magnitude is."

A minority of the students did not interpret the field as uniform, and were therefore categorized as agreeing with the statement

"It might be, it depends on what is happening between the field lines. The field lines might represent a constant field, or one that varies sinusoidally along the horizontal axis for example."

The idea of splitting or merging field lines (LO3) seems to contradict the statement that field lines cannot touch or cross in question 4(a). Nevertheless, many students agreed with the statement in question 4(d), even after disagreeing

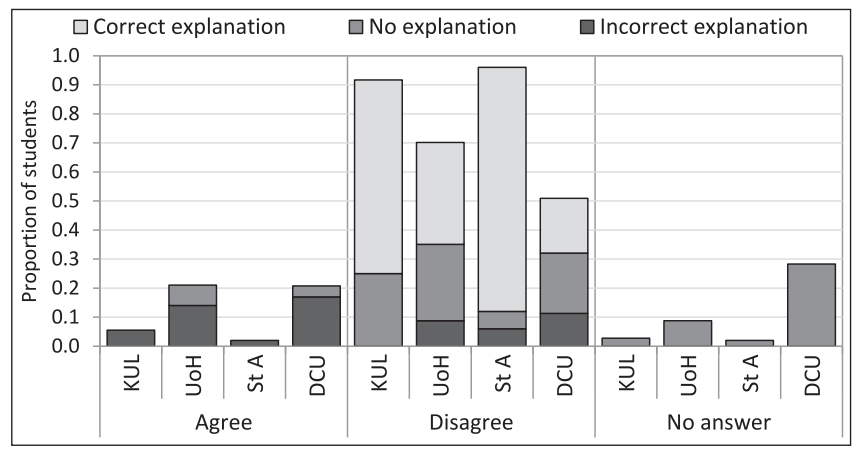

FIG. 9. Distribution of responses and corresponding argumentation to question 4(c) [Fig. 1(d)].

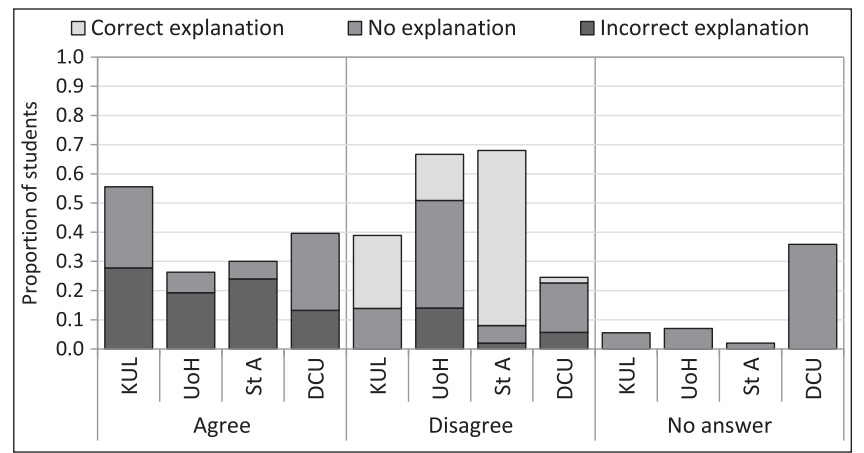

FIG. 10. Distribution of responses and corresponding argumentation to question 4(d) [Fig. 1(d)].

with the statement in question 4(a). When comparing the results in Fig. 10 with those from Fig. 8, it is clear that some students (20\%-30\%) deem it possible that field lines can merge, while explaining correctly why field lines cannot cross.

There where generally two kinds of correct explanations to argue why the statement is incorrect: arguments based on the direction of the field and arguments based on the magnitude of the field at the point where the two field lines merge.

"No, as at the point of intersection the field will be pointing in two slightly different directions at once. Spacing between field lines may decrease to indicate increasing strength but they do not merge."

"When field lines merge, the density of the field is infinite, meaning infinite field strength (not possible physically!)"

Typically, students agreed with the statement when they had incorrect ideas about what field lines represent:

"Yes, when a function converges to a certain value, then field lines will merge."

In some cases, students thought of specific examples that seem to show such behavior:

"True, this happens, for example, at the poles of a magnetic dipole field."

While they may have misinterpreted the images, it is not unlikely that they have encountered inaccurate illustrations in text books or other sources.

\section{Difficulties with setting up a symbolic expression of a vector field}

In questions 2(b) and 3(b) of the VFR test, students were asked to give a possible algebraic expression for the visualizations of the vector fields of Fig. 1. The results 


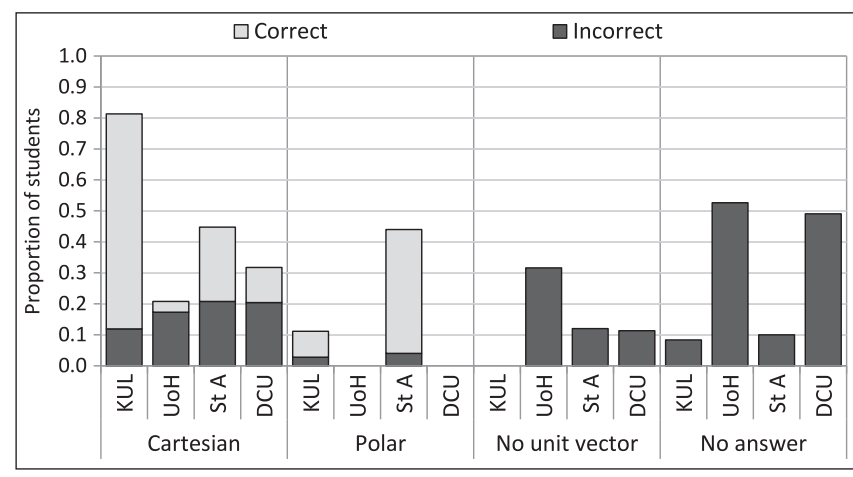

FIG. 11. Proportion of correct responses, depending on the chosen coordinate system, to question 2(b) [Fig. 1(b)].

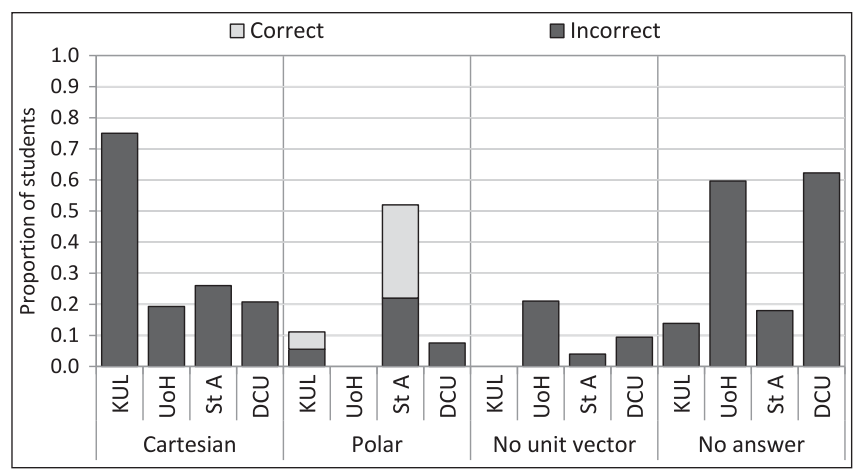

FIG. 12. Proportion of correct responses, depending on the chosen coordinate system, to question 3(b) [Fig. 1(c)].

are presented in Figs. 11 and 12. The considerable fraction of students that produced an incoherent answer or did not respond at all shows that these questions presented a high level of difficulty. Since students had already sketched a corresponding field line diagram and vector field plot for the visualizations in questions 2(a) and 3(a), respectively, and the results for questions 2(b) and 3(b) are comparable, we discuss responses to both questions simultaneously in this section.

The most obvious error is not writing any (unit) vectors and therefore neglecting the direction of the vector field (SD2). In such cases, students typically wrote down equations for the magnitude of the Coulomb force $\left(F=k Q q / r^{2}\right)$ or the magnitude of the electric field of a point charge $\left(E=Q /\left(4 \pi \epsilon_{0} r^{2}\right)\right)$. Other students wrote down expressions that seem to be meaningless $\left(r=\sqrt{x^{2}+y^{2}}\right)$. Such responses were to both questions, indicating that some students are rote learning relations between certain types of fields and mathematical equations without developing a deeper understanding of the physics that is involved.

Students who write unit vectors can choose between Cartesian and polar (or cylindrical) coordinates. The difficulty lies in writing how the field changes in every direction, in order to get both the magnitude (SM1) and direction (SD1) of the field right. It is interesting to note that again some students confused characteristics of field lines and equipotential lines, and, consequently, wrote an incorrect expression for the field. A student who sketched a field line diagram similar to Fig. 7(c) as a response to question 2(a), for example, answered question 2(b) with

$$
\begin{aligned}
& \text { " } \mathbf{v}=x \hat{\mathbf{x}}+y \hat{\mathbf{y}}, \text { and the expression for a circle is } \\
& x^{2}+y^{2}=R^{2} \text { ", }
\end{aligned}
$$

This student seems to write an equation for the given field vector plot (the former) and one for his constructed field line diagram (the latter). Many students who sketched field vectors perpendicular to field lines, or field lines perpendicular to field vectors, were also inconsistent in the algebraic expression that they constructed: sometimes it matched the features of the field line diagram and sometimes it corresponded to the field vector plot. This may indicate that some students were not aware that both visualizations should yield the same mathematical expression.

Taking the symmetry of the fields in question 2(b) and 3(b) into account, polar coordinates may be more appropriate than Cartesian coordinates. However, as can be seen from the results in Figs. 11 and 12, students often focus on the latter. While setting up a correct expression in Cartesian coordinates is still relatively easy for the field in question 2 (e.g., $\mathbf{v}=x \hat{\mathbf{x}}+y \hat{\mathbf{y}})$, it is quite a challenge to do so for the field in question 3 [e.g., $\left.\mathbf{v}=-y /\left(x^{2}+y^{2}\right) \hat{\mathbf{x}}+x /\left(x^{2}+y^{2}\right) \hat{\mathbf{y}}\right]$. Only 1 out of about 60 students who opted for Cartesian coordinates in question 3(b) came close to this equation, but did not write the minus sign.

Examples of correct expressions in polar coordinates are $\mathbf{v}=r \hat{\mathbf{r}}$ and $\mathbf{v}=\frac{1}{r} \hat{\boldsymbol{\theta}}$ for question 2(b) and 3(b), respectively. Again, more students wrote down a correct expression for question 2 than for question 3 . This is explained by the results that were discussed in the previous sections: many students incorrectly deduced from the field line diagram in question 3 that the vector field has a constant or increasing magnitude. Consequently, they wrote an equation that matched their field vector plot in question 3(a), e.g., $\mathbf{v}=r \hat{\boldsymbol{\theta}}$. This was rather unexpected, since the vector field displayed is a familiar example of the magnetic field around a current carrying wire.

It is clear from Fig. 11 and Fig. 12 that students' fluency with writing down a symbolic expression for a vector field when given a graphical representation varies greatly by institution. Nevertheless, the results show that while the majority of students do realize they need to use unit vectors, many students struggle with constructing an expression for the vector field in question 3. It is not surprising that students strongly prefer Cartesian over polar coordinates. However, it is interesting to see that the success rate when using polar coordinates is generally a lot higher. This shows that it may be helpful to focus stronger on when it is 
appropriate to use polar coordinates when teaching about vector fields.

\section{DISCUSSION AND IMPLICATIONS FOR INSTRUCTION}

In this section we discuss the most prevalent student difficulties that were presented in the previous section, some of which were observed in almost every question on the VFR test. Being aware of students' fluency with various representations of vector fields might help teachers to design a more effective learning environment. Therefore, we suggest some implications regarding instruction on the use of vector fields in mathematics and physics courses. In addition, this discussion can serve as the starting point for deeper and more profound research studies aiming to determine what causes the identified student difficulties.

From the results of question 1 on the VFR test, it is clear that many students struggle with vector addition and decomposition when switching from a symbolic to a graphical representation of a vector field. While most students explained correctly that the $x$ direction in question 1 was constant, and the $y$ direction changed with $x$, many sketches did not show this behavior properly. This resulted in drawings in which the vector field had an incorrect or incomplete direction or magnitude. Since vectors are used in so many branches of mathematics and physics, a lot of research has been conducted on vector addition and decomposition [35,48-63]. These studies suggest that students struggle with vector addition across different contexts and levels of instruction. In the context of our questionnaire, it may be possible that most students understand the basics of vector addition when asked to sketch a single vector, but may not know how to use the same principle in the case of a vector field, or confuse vector field components with coordinates $[3,26]$. Since the KU Leuven and St. Andrews students were generally more successful in question 1, our findings imply that students benefit from a prior course that explicitly focuses on switching from algebraic expressions to visualizations of vector fields. This leads us to believe that additional examples and exercises specifically addressing the identified issues may improve students' abilities to construct a vector field plot and field line diagram of a symbolic expression of a vector field. Clearly, such assignments should not be limited to mathematics classes, but can also be invaluable in physics modules, such as electromagnetism.

One of the most prevalent difficulties, seen in almost every question on the test and during both qualitative studies, was the misinterpretation and incorrect sketching of the density of field line diagrams as an indication of the vector field's magnitude. This may be surprising, as it is a key feature of the field line representation. Nevertheless, when looking at common textbooks, we see that field line diagrams are often ambiguously drawn or poorly explained [64]. In addition, these findings agree with those of Albe et al. [29]: the construction of graphical representations of vector fields by students is often badly done, and therefore meaningless. This helps us make sense of the results from our studies regarding students' understanding of divergence and curl in field line diagrams [37-40]. It seems that most students lack representational fluency when interpreting and constructing field line diagrams, and therefore struggle to determine where the divergence or curl is nonzero in such diagrams. Consequently, we think it is important for instructors to explicitly explain how certain illustrations (e.g., the magnetic field of a current carrying wire) match the key features of the field. In the language of Fredlund et al. [36], teachers need to unpack the representations in order to improve students' insight into field line diagrams and field vector plots. Certainly when the representational feature does not match what is being represented, like when less space between field lines corresponds to a higher magnitude [35]. Perhaps this can be done by using various animations, as suggested by some of the studies that were discussed in Sec. I [30-33]. In addition, it may be useful for students to sketch field lines of various fields, including those with a nonzero divergence at every location of the field. In such fields, field lines should emerge from every point in space. In practice, this means that field lines originate from arbitrary locations in a diagram in such a way that they correctly represent the field. However, students may not have a good sense about what kind of information can or should be lost or ignored when constructing a field line diagram. It is also possible that students have the incorrect idea that all field line diagrams of radial vector fields are similar to the classic example of a point charge. Giving additional assignments in which students have to sketch field line diagrams of various fields, including those with a nonzero divergence at all points, might help students to become aware of the strengths and limitations of the field line diagram representation. In addition, it might improve their understanding regarding the vector operators [37-40].

It was surprising to see that at least $20 \%$ of the students seem to confuse characteristics of field lines and equipotential lines. Typically, they sketched arrows perpendicular to the given field lines, or drew lines perpendicular to the given field vectors without an indication of the direction. Some of them did so consistently over the test, for others this depended on the question. While additional research is needed to get insight into the cause of this misunderstanding, we advise instructors to clearly make the distinction during lectures and exercises, for example, by visualizing both field lines and equipotential lines in a single diagram.

While Törnkvist et al. [34] report that the majority of students involved in their research project did not indicate two crossing field lines as erroneous, most of the students who participated in our study correctly stated that field lines are not allowed to touch or cross each other. Nevertheless, many of them struggled to argue why this is a correct 
statement. In addition, about half of the students thought it would be possible for field lines to split, even after stating field lines cannot touch. However, it is interesting to notice that not a single sketch constructed by a student as a response to the first three questions showed such behavior. The inconsistent ideas about the possibility of crossing and merging field lines indicate students struggle to understand what a field line represents. The incorrect idea that field vectors are not allowed to touch or cross is related to this issue, as many students followed the same reasoning as when arguing why field lines cannot cross. Therefore it is important to clearly distinguish between field vectors and field lines, and indicate that field vectors contain information about the point where the arrow originates from (its tail), while field lines are a depiction of the regional character of the field.

This study was mainly concerned with how students interpret and sketch graphical representations. However, we also observed that when students are asked to set up a symbolic expression of a vector field, they strongly focus on Cartesian coordinates. Nevertheless, success rates were higher when a polar coordinate system was used. At this stage, it is not clear whether this is due to a selection effect in which stronger students are more likely to recognize the usefulness of polar coordinates. While further research is needed to validate these claims, it may be helpful to explicitly practice choosing the most appropriate coordinate system when setting up an algebraic expression. While this is an easy task for experts, it might not be as obvious for students.

\section{CONCLUSIONS AND POSSIBILITIES FOR FURTHER RESEARCH}

We have investigated what difficulties students encounter regarding graphical and symbolic representations of vector fields using both qualitative and quantitative approaches. Based on experiences with individual student interviews and in-class activities, we designed the VFR test, a valid and reliable instrument to probe students' understanding of vector field representations. The results from this study allow us to answer the research questions that were posed in the introduction.

We have observed that students struggle with various characteristics of field vector plots and field line diagrams when constructing visualizations of vector fields. A complete overview of these difficulties can be found in Table I. When asked to sketch field vector plots, students often only drew one set of vectors or even a single arrow. The field line density in students' sketches often did not correspond to the magnitude of the field. In addition, students did not pay a lot of attention to where field lines should start or end. Finally, some students confused field lines with equipotential lines.
When switching from a symbolic to a graphical representation of a vector field, students seemed to struggle with vector addition, resulting in incorrect field vector plots. When setting up a symbolic expression themselves, students preferred a Cartesian coordinate system above a polar one, despite getting a higher success rate when using the latter.

While the prevalence of errors depended strongly on the institution, the difficulties mentioned above were seen at all four universities. This shows the identified difficulties are omnipresent. This calls for more in-depth research studies focusing on the causes of and solutions to these problems.

In our earlier work [37-40], we discussed how students struggle with determining where the divergence or curl is nonzero in graphical representations of vector fields. In general, we assumed student difficulties were mostly related to a limited understanding of the meaning of divergence and curl in visualizations of vector fields. The results from this paper show that students may not only exhibit such difficulties because they lack an understanding of the vector operators, but also because of an insufficient fluency with the corresponding diagrams. Therefore, instructional materials regarding these topics should focus on teaching both how to interpret field vector plots and field line diagrams, and how to determine where the divergence or curl is nonzero in such visualizations.

As mentioned in Sec. I, we have focused exclusively on students' representational fluency with interpreting and constructing field vector plots, field line diagrams, and symbolic expressions of vector fields. However, it might be important to also study their representational flexibility. Our qualitative studies suggest that students may prefer field line diagrams to field vector plots when solving problems regarding vector fields, but additional research is needed to determine whether these findings are generalizable. By investigating students' representational flexibility, we may also learn to what extent they confuse characteristics of field lines and field vectors when visualizing vector fields.

In addition to research opportunities that aim to gain insight into difficulties regarding vector field representations, it may also be interesting to investigate to what extent these difficulties influence students' understanding of physics. Field line diagrams and field vector plots are often used to illustrate gravitational and electromagnetic fields, streamlines in fluid dynamics, or wind velocities. Physics students may therefore benefit from an improved understanding of both graphical and symbolic representations of vector fields.

\section{ACKNOWLEDGMENTS}

We would like to thank all participating students whose cooperation made this research project possible. 
[1] This convention is used in all textbooks that are typically used in introductory physics and mathematics courses.

[2] D. Rosengrant, E. Etkina, and A. Van Heuvelen, An overview of recent research on multiple representations, AIP Conf. Proc. 883, 149 (2007).

[3] J. F. Wagner, C. A. Manogue, and J. R. Thompson, Representation issues: Using mathematics in upper-division physics, AIP Conf. Proc. 1413, 89 (2012).

[4] J. I. Heller and F. Reif, Prescribing effective human problem-solving processes: Problem description in physics, Cognit. Instr. 1, 177 (1984).

[5] A. Van Heuvelen, Learning to think like a physicist: A review of research-based instructional strategies, Am. J. Phys. 59, 891 (1991).

[6] R. J. Dufresne, W. J. Gerace, and W. J. Leonard, Solving physics problems with multiple representations, Phys. Teach. 35, 270 (1997).

[7] P. C.-H Cheng, Unlocking conceptual learning in mathematics and science with effective representational systems, Comput. Educ. 33, 109 (1999).

[8] A. Van Heuvelen and X. Zou, Multiple representations of workenergy processes, Am. J. Phys. 69, 184 (2001).

[9] D. Rosengrant, Case Study: Students' Use of Multiple Representations in Problem Solving, AIP Conf. Proc. 818, 49 (2006).

[10] C. J. De Leone and E. Gire, Is instructional emphasis on the use of non-mathematical representations worth the effort?, AIP Conf. Proc. 818, 45 (2006).

[11] P. B. Kohl and N. D. Finkelstein, Effect of instructional environment on physics students' representational skills, Phys. Rev. ST Phys. Educ. Res. 2, 010102 (2006).

[12] J. F. Wagner, Transfer in Pieces, Cognit. Instr. 24, 1 (2006).

[13] S. Ainsworth, The functions of multiple representations, Comput. Educ. 33, 131 (1999).

[14] S. Ainsworth, P. Bibby, and D. Wood, Examining the Effects of Different Multiple Representational Systems in Learning Primary Mathematics, J. Learn. Sci. 11, 25 (2002).

[15] S. Ainsworth, The educational value of multiplerepresentations when learning complex scientific concepts, in Vis. Theory Pract. Sci. Educ., edited by J. K. Gilbert, M. Reimer, and M. Nakhleh (Springer, Netherlands, 2008) pp. 191-208.

[16] R. Even, Factors involved in linking representations of functions, J. Math. Behav. 17, 105 (1998).

[17] M. De Cock, Representation use and strategy choice in physics problem solving, Phys. Rev. ST Phys. Educ. Res. 8, 020117 (2012).

[18] A. A. Nistal, W. Dooren, G. Clarebout, J. Elen, and L. Verschaffel, Conceptualising, investigating and stimulating representational flexibility in mathematical problem solving and learning: a critical review, ZDM Int. J. Math. Educ. 41, 627 (2009).

[19] A. A. Nistal, W. V. Dooren, and L. Verschaffel, What counts as a flexible representational choice? An evaluation of students' representational choices to solve linear function problems, Instr. Sci. 40, 999 (2012).

[20] K. N. Bieda and M. J. Nathan, Representational disfluency in algebra: Evidence from student gestures and speech, ZDM-Int. J. Math. Educ. 41, 637 (2009).
[21] P. B. Kohl and N. D. Finkelstein, Patterns of multiple representation use by experts and novices during physics problem solving, Phys. Rev. ST Phys. Educ. Res. 4, 010111 (2008).

[22] D.-H. Nguyen and N. Sanjay Rebello, Students' Difficulties in Transfer of Problem Solving Across Representations, AIP Conf. Proc. 1179, 221 (2009).

[23] D. H. Nguyen, E. Gire, and N. Sanjay Rebello, Facilitating students' problem solving across multiple representations in introductory mechanics, AIP Conf. Proc. 1289, 45 (2010).

[24] D. H. Nguyen, E. Gire, and N. Sanjay Rebello, Facilitating strategies for solving work-energy problems in graphical and equational representations, AIP Conf. Proc. 1289, 241 (2010).

[25] D.-H. Nguyen and N. Sanjay Rebello, Students' Difficulties with Multiple Representations in Introductory Mechanics, US-China Educ. Rev. 8, 559 (2011).

[26] E. Gire and E. Price, Graphical representations of vector functions in upper-division E\&M, AIP Conf. Proc. 1413, 27 (2012).

[27] Y. Cao and B. M. Brizuela, High school students' representations and understandings of electric fields, Phys. Rev. Phys. Educ. Res. 12, 020102 (2016).

[28] M. Sağlam and R. Millar, Upper high school students' understanding of electromagnetism, Int. J. Sci. Educ. 28, 543 (2006).

[29] V. Albe, P. Venturini, and J. Lascours, Electromagnetic Concepts in Mathematical Representation of Physics, J. Sci. Educ. Technol. 10, 197 (2001).

[30] J. W. Belcher and R. Mark Bessette, Using 3D animation in teaching introductory electromagnetism, Comput. Graph. (ACM). 35, 18 (2001).

[31] J. W. Belcher and S. Olbert, Field line motion in classical electromagnetism, Am. J. Phys. 71, 220 (2003).

[32] Y. J. Dori and J. W. Belcher, How Does TechnologyEnabled Active Learning Affect Undergraduate Students' Understanding of Electromagnetism Concepts?, J. Learn. Sci. 14, 243 (2005).

[33] R. G. Sousa, P. J. V. Garcia, V. Marinho, and A. Mouraz, Visualization of electric field lines in an engineering education context, in 1st Int. Conf. Port. Soc. Eng. Educ. (IEEE, New York City, 2013), pp. 1-6.

[34] S. Törnkvist, K.-A. Pettersson, and G. Tranströmer, Confusion by representation: On student's comprehension of the electric field concept, Am. J. Phys. 61, 335 (1993).

[35] E. Gire and E. Price, Arrows as anchors: An analysis of the material features of electric field vector arrows, Phys. Rev. ST Phys. Educ. Res. 10, 020112 (2014).

[36] T. Fredlund, C. Linder, J. Airey, and A. Linder, Unpacking physics representations: Towards an appreciation of disciplinary affordance, Phys. Rev. ST Phys. Educ. Res. 10, 020129 (2014).

[37] L. Bollen, P. van Kampen, and M. De Cock, Students' difficulties with vector calculus in electrodynamics, Phys. Rev. ST Phys. Educ. Res. 11, 020129 (2015).

[38] L. Bollen, P. van Kampen, C. Baily, and M. De Cock, Qualitative investigation into students' use of divergence and curl in electromagnetism, Phys. Rev. Phys. Educ. Res. 12, 020134 (2016). 
[39] C. Baily, L. Bollen, A. Pattie, P. van Kampen, and M. De Cock, Student thinking about the divergence and curl in mathematics and physics contexts, Proceedings of the Physics Education Research Conference, College Park, MD, 2015 (AIP, New York, 2015), pp. 51-54.

[40] C. Baily and C. Astolfi, Student Reasoning about the Divergence of a Vector Field, Proceedings of the Physics Education Research Conference, College Park, MD, 2014 (AIP, New York, 2015), pp. 31-34.

[41] D. C. Giancoli, Physics for Scientists and Engineers with Modern Physics, , 4th ed. (Addison-Wesley, Reading, MA, 2008), p. 1328.

[42] R. A. Adams and C. Essex, Calculus: A Complete Course, 8th ed. (Pearson Education, 2013), p. 1136.

[43] D. Halliday, R. Resnick, and J. Walker, Principles of Physics, 9th ed. (John Wiley and Sons, New York, 2011), p. 1136.

[44] W. J. Duffin, Electricity and Magnetism, 4th ed. (McGrawHill, New York, 1990), p. 437.

[45] H. D. Young and R. A. Freedman, University Physics with Modern Physics, 14th ed. (Pearson, New York, 2015), p. 1600 .

[46] J. Cohen, A Coefficient of Agreement for Nominal Scales, Educ. Psychol. Meas. 20, 37 (1960).

[47] A. J. Viera and J. M. Garrett, Understanding Interobserver Agreement: The Kappa Statistic, Fam. Med. 37, 360 (2005).

[48] D. Hestenes, M. Wells, and G. Swackhamer, Force Concept Inventory, Phys. Teach. 30, 141 (1992).

[49] R. D. Knight, The vector knowledge of beginning physics students, Phys. Teach. 33, 74 (1995).

[50] N.-L. Nguyen and D. E. Meltzer, Initial understanding of vector concepts among students in introductory physics courses, Am. J. Phys. 71, 630 (2003).

[51] S. Flores, S. E. Kanim, and C. H. Kautz, Student use of vectors in introductory mechanics, Am. J. Phys. 72, 460 (2004).

[52] J. Van Deventer and M. C. Wittmann, Comparing student use of mathematical and physical vector representations, AIP Conf. Proc. 951, 208 (2007).

[53] J. Van Deventer, Ph.D. thesis, University of Maine, 2008.

[54] J. M. Hawkins, J. R. Thompson, and M. C. Wittmann, Students Consistency of Graphical Vector Addition
Method on 2-D Vector Addition Tasks, AIP Conf. Proc. 1179, 161 (2009).

[55] P. Barniol and G. Zavala, Vector Addition: Effect of the Context and Position of the Vectors, AIP Conf. Proc. 1289, 73 (2010).

[56] J. M. Hawkins, J. R. Thompson, M. C. Wittmann, E. C. Sayre, and B. W. Frank, Students' responses to different representations of a vector addition question, AIP Conf. Proc. 1289, 165 (2010).

[57] U. Wutchana and N. Emarat, Students' Understanding of Graphical Vector Addition in One and Two Dimensions, Eurasian J. Phys. Chem. Educ. 3, 102 (2011).

[58] P. Barniol and G. Zavala, Testing Students' Understanding of Vector Concepts, Proceedings of the Physics Education Research Conference 2013, Portland, OR (AIP, New York, 2014), pp. 65-68.

[59] L. Doughty, Ph.D. thesis, Dublin City University, 2013.

[60] P. Barniol and G. Zavala, Test of understanding of vectors: A reliable multiple-choice vector concept test, Phys. Rev. ST Phys. Educ. Res. 10, 010121 (2014).

[61] P. Barniol and G. Zavala, Students' difficulties in problems that involve unit-vector notation, Lat. Am. J. Phys. Educ. 8, p4403-1 (2014).

[62] A. F. Heckler and T. M. Scaife, Adding and subtracting vectors: The problem with the arrow representation, Phys. Rev. ST Phys. Educ. Res. 11, 010101 (2015).

[63] P. J. Emigh, G. Passante, and P. S. Shaffer, Student Understanding of Superposition: Vectors and Wave Functions, Proceedings of the Physics Education Research Conference 2016, Sacremento, CA (AIP, New York, 2016), pp. 112-115.

[64] This is a general impression we get from looking at physics and mathematics textbooks, for example, when the density of magnetic field lines around a current carrying wire does not clearly decrease with increasing distance from the wire (Griffiths' textbook [65], 3rd ed., Fig. 5.3.) or when Earth's gravitational field lines only start at the surface of Earth (Giancoli's textbook [41], 4th ed., Figs. 21-35). This does not mean that these textbooks should not be used. It rather shows that a well thought-out complementary instruction strategy is invaluable.

[65] D. J. Griffiths, Introduction to Electrodynamics, 4th ed. (Addison-Wesley, Reading, MA, 2012), p. 624. 